\title{
Gestión de conocimiento: una apuesta por la calidad institucional ${ }^{*}$
}

\author{
Luis Alberto Sarmiento S."* \\ Recibido: 20 de enero de 2016 • Revisado: 7 de febrero de 2016 \\ Aprobado: 22 de febrero de 2016
}

\section{Resumen}

Considerando la necesidad inminente de re-dimensionar el conocimiento y su reconocimiento como factor determinante en las instituciones de educación superior, la gestión del conocimiento (GC) se presenta como aquella alternativa interesante que ejecuta tan difícil tarea. Al hacer un análisis riguroso del sentido del conocimiento, los horizontes de comprensión se abren y disponen una serie de competencias que hacen más atractivo el impacto del saber que se produce en el escenario académico. Por tanto, el conocimiento deja de ser valor agregado y casi opcional en las IES y se hace factor de producción llevando a la formación de la competencia investigativa.

Palabras claves: Conocimiento, investigación, gestión del conocimiento (GC), cultura del conocimiento, instituciones de educación superior (IES).

"El artículo es producto del proyecto de investigación institucional de la Corporación Universitaria Republicana: “Gestión del Conocimiento". Dicho proyecto de investigación fue financiado por la citada Academia.

* Licenciado en Educación Religiosa - Universidad de la Salle. Magíster en Educación - Pontificia Universidad Javeriana. Docente de la Corporación Universitaria Republicana y Director Académico en el Liceo Hermano Miguel la Salle. Correo electrónico: lualsa23@gmail.com. 


\title{
Knowledge Management: A WAGER FOR INSTITUTIONAL QUALITY
}

\begin{abstract}
Considering the imminent need of changing the way of perceiving knowledge and its relevance in the higher education, the knowledge management (KM) appears as an interesting alternative that can carry on this hard task. After having analysing firmly the real sense of knowledge, the understanding competence come out and with it appears also a wide range of competences that make the impact of learning that occurs in the academic scenario more attractive. Therefore, knowledge ceases to be an added value or an option in the higher education. On the contrary, it has become in a vital factor that promotes the research competence.
\end{abstract}

Keywords: Knowledge, research, knowledge management (KM), knowledge culture, higher education institutions (HEI).

\section{Conhecimento: uma APOSTA PELA QUALIDAde institucional}

\section{Resumo}

Considerando a necessidade iminente para redimensionar o conhecimento e seu reconhecimento como um fator determinante nas instituições de ensino superior, a gestão do conhecimento (GC) apresenta-se como aquela alternativa interessante que executa tão difícil tarefa. Ao fazer uma análise rigorosa do significado do conhecimento, os horizontes de compreensão são abertos e dispõem uma gama de habilidades que tornam mais atraente o impacto do conhecimento produzido no ambiente acadêmico. Portanto, o conhecimento deixa de ser o valor acrescentado e quase opcional nas IES e se fez fator de produção, levando à formação da competência de pesquisa.

Palavras-chave: Conhecimento, pesquisa, gestão do conhecimento (GC), cultura do conhecimento, instituições de ensino superior (IES). 


\section{Una apuesta por la calidad institucional}

La función social de la universidad se ha visto fluctuar entre intenciones educativas establecidas y proyectos políticos particulares. Es en este sentido que resulta procedente afirmar que

La universidad se encuentra permeada por prácticas burocráticas y neoliberales que le han convertido en el espacio ideal para atender las demandas del sector empresarial y garantizar la oferta de paquetes educativos que, muchas veces, son creados sin rigor, calidad ni pertinencia. Así, es común encontrar cómo programas de formación universitarios responden más a una "necesidad" de negocio y prestigio institucional, así como a la certificación de mano de obra que pueda vincularse a las empresas. No es de extrañar que el conocimiento propicio en las universidades quede al servicio de las disposiciones externas o, en el peor de los casos, postrado ante los reconocimientos, individuales y económicos, de los académicos y/o investigadores. (Aferreyra, 2013, p. 4)

Siendo las instituciones de educación superior epicentros de conocimiento objetivo, su valor no puede verse reducido a formas pobres de producción de éste. La identidad universitaria se enmarca en el valor que se le otorgue al conocimiento; el resto de dinámicas institucionales están al tanto de apoyar y promover la investigación. En sectores industrializados, la promoción de la acción investigativa deja entrever el interés por la promoción científica en cualquiera de las áreas del saber; desafortunadamente la oferta educativa de los no industrializados - al contrario- está dirigida a sectores de la sociedad particulares.

La labor académica propende por la cualificación de la sociedad y su valor está dado en la capacidad de transformación social que se instaure; esto se hace también con investigación objetiva y acción participativa de los centros de conocimiento superior. De allí que el interés que venga por parte de los gobiernos redunde en beneficios para su Estado, así como para la posibilidad de competitividad mundial de acuerdo a los avances científicos que se adelanten. Así lo afirman las bases de un plan de acción para la adecuación del sistema de propiedad intelectual a la competitividad y productividad nacional cuando dice que 
El conocimiento es en el siglo XXI un recurso crucial entre los múltiples determinantes de la competitividad de un país. La generación de valor con base en la creación intelectual, es decir, la producción de conocimiento, así como el uso del conocimiento disponible, son herramientas fundamentales para producir bienes y servicios innovadores capaces de insertarse adecuadamente en mercados competitivos. (ODAI, 2008)

En esta perspectiva, la comprensión que se tenga de conocimiento en el marco de las instituciones de educación superior es determinante y de ella dependerán los horizontes de comprensión que la misma decida. Comúnmente se ha entendido el conocimiento como aquella construcción objetiva, validada de manera rigurosa por distintos métodos y que designa leyes, teorías y reglas para entender el funcionamiento natural y social. Sin embargo, este tipo de concepciones muestran la punta del iceberg escondiendo tras de sí, procedimientos, intereses y comprensiones que pueden determinar nuevos caminos hermenéuticos para la explicación de fenómenos de toda índole o naturaleza. Será entonces necesario indagar por las concepciones que las instituciones de educación superior tengan sobre conocimiento para profundizar por fin, en las implicaciones que trae hacer gestión del conocimiento.

$\mathrm{Al}$ entenderse como factor de producción, tangencialmente se sospecha de las características socioculturales en las que se desarrolla la concepción, sin embargo, es factor productivo en tanto que, desde las directrices organizacionales, concentra la actividad competitiva de la institución y la dispone en un escenario de interacción académica. De esta manera, el conocimiento configura el interés científico al trascender del cerrado círculo científico al espacio cotidiano de la esfera social. E1 conocimiento se hace tangible al permear los espacios de producción académica de la universidad; esto es, ya no es de sujetos particulares sino que se vuelve un objeto per se asequible y administrable por toda la comunidad educativa.

En este contexto, el conocimiento en las instituciones de educación superior será evidencia de capitalización de información y su valor estará dado por la intención que a éste se le otorgue. En otras palabras, el conocimiento es aquella apuesta definitiva que las instituciones de educación superior hacen para traducir su trayectoria en términos de avance y producción científica en cualquiera de las áreas en la que se pretenda formar. Puede entonces ahora profundizarse en el establecimiento del conocimiento como tarea fundamental de la universidad, para lo cual el Consejo nacional de educación superior sostiene: 
Las funciones tradicionales que se consideran propias de la educación superior: formación; investigación y extensión/proyección social, se constituyen en referente para que toda la institución defina prioridades, sin excluir ninguna de las tres, y en ellas caracterice su propuesta educativa, en los diferentes niveles de formación: técnica, tecnológica, profesional, de investigación. En el concepto mundialmente aceptado, la universidad como institución de educación superior, organiza la formación, predominantemente, a partir y como resultado de los procesos de indagación e investigación, y por lo tanto en función prioritaria de la exploración, construcción, difusión y aplicación del conocimiento. (2013, p. 4)

La investigación contribuye enormemente a la construcción de concepto de conocimiento que la institución pretenda debido a su enorme preocupación por el avance científico riguroso, serio y sistemático. Considerando el proceso investigativo como de enorme riqueza para la IES, no puede existir producción de conocimiento sin trabajo investigativo. En lo referente, por ejemplo, a la lectura social que hace toda institución académica no puede negarse la importancia de la tecnología y su influencia en todos los sectores de la sociedad, por ello, entiéndase también como avance científico la consolidación tecnológica y todo tipo de avances que por la investigación pueda realizarse.

Suele suceder que todo intento investigativo -o pseduoinvestigación- sea asumido como conocimiento, haciendo que cualquier tipo de fuente sea confiable y por ende competitiva al momento de hacer ciencia. Error garrafal es éste y su causa principal es la carencia de recorridos investigativos rigurosos. Confundir información y conocimiento es en la actualidad bastante tentador ya por la posibilidad de fácil acceso, ya por la emancipación de nuevas formas de comunicación. ¿Qué hacer al respecto?

La ciencia de la información, surge a mediados del siglo XX como paradigma interdisciplinario que se ocupa de aspectos relacionados con la producción, colección, organización, difusión, recuperación y optimización del conocimiento, independientemente del soporte en el que se encuentre plasmado (Múnera, 2012). Al respecto Taylor (1996) la concibe como:

La ciencia que investiga las propiedades y el comportamiento de la información, las fuerzas que gobiernan su flujo y los medios para procesarlas para acceso y uso óptimo. El proceso incluye la generación y diseminación, recolección, organización, almacenamiento, recuperación, interpretación y uso de la información. El campo 
se deriva o se relaciona con la matemática, la lingüística, la psicología, la tecnología de la computación, la investigación de operaciones, las artes gráficas, la comunicación, la bibliotecología, la administración y algunos otros campos. (pp. 15-28)

Su concepción algo técnica de lo que es la ciencia de la información, hace de ella un recurso indispensable para la competencia en las disciplinas descritas. Sin embargo, puede haber complemento con la concepción que presenta Borko (1968) cuando afirma que es una:

Disciplina que investiga las propiedades y el comportamiento de la información, las fuerzas que rigen su flujo y los métodos para procesarla, a fin de obtener accesibilidad óptimas. Está interesada en un conjunto de conocimientos relacionados con el origen, colección, organización, almacenaje, recuperación, interpretación, transmisión, transformación y utilización de información. (pp. 3-5)

Un acento casi pragmatista dado por Borko, evidente en su definición, respalda la concepción del elemento informativo como aquello práctico y de fácil acceso, dado por fuentes y resultado de un proceso subjetivo. Sin el ánimo de satanizar la expresión, la información es solo un momento previo al conocimiento y su objetividad depende del uso que a ésta se le dé. No todo recurso informativo resulta en conocimiento científico. Fueron los primeros filósofos los que, ya previniendo cualquier tipo de ambigüedad al respecto, distinguieron entre el conocimiento científico (episteme) y el conocimiento vulgar (doxa). Así pues, la ciencia de la información contribuye al enriquecimiento sistemático de lo que se convierte en primer filtro para hacer conocimiento.

Ahora bien, hecha la conceptualización de los elementos constitutivos de la información, el conocimiento se presenta como su elemento revelador y el resultado objetivo de un proceso consultivo, organizativo, sistemático, comprobable y sujeto de confrontación. No es distinto a la información pero sí es su estado pleno. Pavez conceptualiza el conocimiento como

Las creencias cognitivas, confirmadas, experimentadas y contextuadas del conocedor sobre el objeto, las cuales estarán condicionadas por el entorno, y serán potenciadas y sistematizadas por las capacidades del conocedor, las cuales establecen las bases para la acción objetiva y la generación de valor. (2001, p. 15) 
Podría concluirse este aparte, acerca de la definición del conocimiento, con el aporte de Berrocal y Pereda (2001) diciendo:

El conocimiento es algo más: es un conjunto de informaciones y de experiencias estructuradas, de valores y de información contextual que permite cambiar la forma de actuar del receptor. El conocimiento, por tanto, incluiría tanto el «saber», como el «saber hacer» y el «saber estar», incluidos en el concepto de competencia; esto es, los conocimientos teóricos sobre un tema determinado, la aplicación de los mismos a la resolución de los problemas prácticos del trabajo, y las actitudes que facilitan un comportamiento acorde con los valores y la cultura de la organización. (p. 644)

Así entonces, las consideraciones necesarias para entrar en materia sobre el proceso de gestión de conocimiento (GC) están hechas: ya se soslayó el contexto donde se pretende hacer GC, posteriormente se hicieron las consideraciones necesarias del elemento conocimiento en una institución de educación superior (IES), destacando la diferencia fundamental entre información y conocimiento y su apoyo necesario en la ciencia de la información. Entonces, para articular el recorrido conceptual anterior con la gestión del conocimiento es propicio consolidar el horizonte de comprensión desde donde se aborda la GC y así aterrizar la teoría a la práctica en la Corporación Universitaria Republicana.

Por gestión del conocimiento se entiende una

Nueva tendencia administrativa que surge a finales del siglo XX, como una forma de optimizar los recursos de una institución desde el aprovechamiento eficiente del conocimiento. En términos generales, se asume a la Gestión del Conocimiento como la capacidad que tiene una organización de generar valor con base en el conocimiento. (Múnera, 2012, p. 60)

Es el conocimiento entendido como potencial de desarrollo, factor de desarrollo y oportunidad de competencia.

Desde sus inicios, la GC se ha propuesto dar una explicación sistemática al recurso informativo con el que cuenta una institución académica sobre todo si se trata de una IES. Y ha hecho que todo este tipo de instituciones encuentren lo importante que es direccionar su preocupación hacia la producción de conocimiento. 
Vale la pena hacer hincapié en este factor ya que la generación de nuevos conocimientos es considerada como la primera y única tarea de la IES. Siendo ésta última una organización del conocimiento, su progreso depende de la capitalización de la información y del conocimiento.

Se podría adoptar la definición de gestión de conocimiento que da Aledo: "La gestión del aprendizaje, obtenido en un momento y lugar determinado, para permitir trasladarlo y aplicarlo a otro lugar y momento, para obtener los resultados esperados en menos tiempo y con menos errores" (1998, p. 32). La referencia a la GC es hoy determinante en tanto que ya perdió su carácter opcional. Es una obligación de la institución de educación superior el tenerla si quiere sobrevivir en el mundo académico.

Las organizaciones se encuentran ante el reto de innovar o morir. La innovación exige aprendizaje y desarrollo de nuevos conocimientos; como consecuencia, las competencias fundamentales para la supervivencia de la organización van a ser las referidas a su capacidad para adquirir conocimientos, para adaptarse y cambiar, incluso adelantándose a los cambios. (Berrocal y Pereda, 2001, p. 643)

Se considera que la teoría de la gestión del conocimiento considera tres áreas de reflexión. A saber: la identificación de prioridades de investigación, el estudio del capital intelectual y de los intangibles de las instituciones, y la proyección de la universidad en su entorno para propiciar la apropiación del conocimiento. Cada una de estas está asociada a las políticas investigativas de la Corporación y mantienen como gran interés la configuración del conocimiento como agente transformador. Por lo mismo, se hará necesario reforzar y, en otros casos, modificar las prácticas pedagógicas en pro de la instalación del conocimiento como cultura institucional.

La Universidad siempre se ha caracterizado por cumplir la función de descubrir, transmitir, aplicar, conservar y superar el conocimiento, en un intento por realizar su función en independencia de los factores de poder humano (económico, político o religioso), ha podido adaptarse a las condiciones y exigencias sociales de cada tiempo y ha sido el foco de origen de los más grandes desarrollos tecnológicos, científicos, humanísticos, en otras palabras, la continua investigación, educación y revaluación de conocimiento que genera innovación ha sido y será su aporte a 
la comunidad, tal vez siendo más sobresaliente ahora que se está evolucionando dentro de una sociedad de conocimiento. (Pérez y Amaya, 2013, p. 67)

Al respecto, se considera que las universidades, en su proyecto educativo institucional, deberían establecer como misión

La preparación integral de nuestros educandos, mediante estrategias participativas de enseñanza, aprendizaje y formación, que integren la docencia, la investigación y la extensión, dentro de claros principios humanísticos, éticos y ecológicos, para viabilizar el progreso individual y colectivo, en todos los campos del saber, acordes con el carácter institucional legalmente reconocido. (Corporación Universitaria Republicana, 2013, pp. 12-13)

Lo anterior conlleva a que las universidades asuman un proceso de autorreconocimiento en perspectiva al GC y renueve, en tal caso, procesos curriculares, toda vez que se pretenda llegar a una sólida cultura de conocimiento.

\section{Conclusiones}

La gestión del conocimiento es un alternativa de reconocimiento institucional bastante interesante, sobre todo para el contexto social, cultural y académico que rodea a la Academia.

Es importante entender el conocimiento como un paradigma productivo y posibilidad determinante de competencia.

En aras de lograr la consolidación de las Universidades en el marco de la investigación, es necesario cumplir aquellas condiciones para que la GC se establezca en una organización. Estas podrán tenerse en cuenta al momento de hacer seguimiento y control de lo que se ha llamado cultura del conocimiento. Las condiciones son las siguientes:

1. El conocimiento se da en el proceso de relación entre el ser humano y su entorno.

2. En ese proceso interviene tanto la experiencia de percepción del mundo material a través de los sentidos, como el análisis y la abstracción por la 
mente humana. Es decir, el conocimiento es un continuum entre la práctica y la teoría, entre la experiencia y la razón, entre la materia y la consciencia.

3. La duda, la indagación, la fragilidad de cualquier "verdad" científica y la curiosidad se constituyen en estímulos del deseo permanente del ser humano por el conocimiento.

4. Existen diferentes marcos teóricos y metodológicos para el proceso de conocimiento, pero el establecimiento de relaciones (de semejanza, de oposición, de cantidad, de cualidad, de causalidad, entre otras) es el mecanismo básico con el que operamos los seres humano en ese proceso.

\section{Referencias}

Aferreyra, A. (2013). La Política Educativa, más allá del concepto. Recuperado dehttp:// horacioaferreyra.com.ar/wp-content/uploads/2014/02/LA-POL\%C3\%8DTICAEDUCATIVA-.pdf

Aledo, A. (1998). La gestión del conocimiento en el grupo Heineken (Heineken Uni- versity). Training and Development Digest (pp. 28-31).

Borko, H. (1968). Information Science. What is it? American Documentation (pp. 3-5). v. 19, n. 1. Corporación Universitaria Republicana. (2013) "Proyecto educativo institucional" Bogotá: Autor.

Consejo Nacional de Educación Superior. (2013). Rediseño del esquema de gestión de las regalías estaría en propuesta de política pública del CESU. Recuperado de http:// universidad.edu.co/index.php?option $=$ com_content\&view $=$ article $\&$ id $=4315 \% 3$ Aredise no-del-esquema-de-gestion-de-las-regalias-estaria-en-propuesta-de-politica-publicadel-cesu\&catid=16\%3Anoticias\&Itemid=198

Múnera, M. (2012). Ciencia de la información y gestión del conocimiento. Perspectivas em Gestão \& Conhecimento (pp. 57-64).

ODAI. (2008). ODAI forma parte de las estrategias definidas por el CONPES de propiedad intelectual en Colombia. Recuperado de http://odai.org/2008/07/odai-forma-parte-delas-estrategias-definidas-por-el-conpes-de-propiedad-intelectual-en-colombia/

Pavez, A. (2001). Modelo de implantación de gestión de la información para la generación de ventajas competitivas. Tesis de Ingeniería informática. Valparaíso: Universidad Técnica Federico Santa María.

Pereda, S. y Berrocal, F. (2001). Gestión de recursos humanos por competencias. Madrid: Centro de Estudios Ramón Areces (1. ${ }^{\mathrm{a}}$ reimpresión). 
Pérez, A., Amaya, R. y Barceló, M. (2013). A methodology for the identification of key knowledge to improve decision making in the training area. The IUP Journal of Knowledge Management.

Taylor, R. S. (1996). Professional aspects of Information Science and Technology (pp. 15-28). Annual Review of Information Science Tecnology, V. 1. 